\title{
Spurious Radiation from Microstrip Interconnects
}

\author{
M. I. Aksun and Raj Mittra, Fellow, IEEE
}

\begin{abstract}
The level of spurious radiation from microstrip interconnects, which are modeled here as either single or asymmetric parallel microstrip lines terminated by arbitrary complex load impedances, is investigated in this paper. The calculation of the spurious radiation requires a knowledge of the current distributions on the microstrip lines, and the first step is to compute these distributions efficiently. This is carried out here by using the method of moments in conjunction with closedform spatial domain Green's functions that circumvent the need for time-consuming evaluation of Sommerfeld integrals. Once the current distributions on the etches have been obtained, the level of spurious radiation, which is defined as the radiated power crossing the plane parallel to the plane of interconnects, is calculated. The dependence of the spurious radiation on the lengths of the lines and on the termination impedances of the etches is also studied.
\end{abstract}

\section{INTRODUCTION}

$\mathbf{T}$ HE estimation of spurious radiation from microstrip interconnects, and a systematic design of the layouts of these interconnects ensuring that the radiation level is below the specifications set by the regulatory agencies, are important problems that arise in the electrical design of the packages for digital and communication circuits. One approach to estimating the spurious radiation level is to use static or quasi-static approximations and calculate the capacitance and inductance parameters [1] of the interconnect configuration. The current distribution of the etches is obtained next and the spurious radiation is computed by using the current distribution. While the procedure just outlined is frequently used in practice, it does not adequately take into account the dispersive nature of etches, or the retardation phenomenon, which might have a significant effect on the result. The rigorous analysis of printed circuit elements, such as microstrip interconnects terminated by complex loads, microstrip discontinuities, patch antennas and printed dipoles (see Fig. 1), requires the use of the vector and scalar Green's functions for a substrate layer backed by a ground plane [2]. It is well known that the Green's functions for microstrip geometries are improper integrals [3], also called Sommerfeld integrals, whose integrands are oscillatory and slowly decaying functions; hence, their calculation is very time-consuming for many practical configurations of interest.

Manuscript received April 21, 1992; revised September 30, 1992. The work was sponsored in part by Joint Services Electronics Program under Grant N00014-90-J-1270.

M. I. Aksun was formerly with the Electromagnetic Communication Laboratory, Department of Electrical and Computer Engineering, University of Illinois at Urbana-Champaign, Urbana, IL 61801. He is currently with the Department of Electrical and Electronics Engineering, Bilkent University, 06533 Ankara, Turkey.

R. Mittra is with the Electromagnetic Communication Laboratory, Department of Electrical and Computer Engineering, University of Illinois at Urbana-Champaign, Urbana, IL 61801.

IEEE Log Number 9206658.

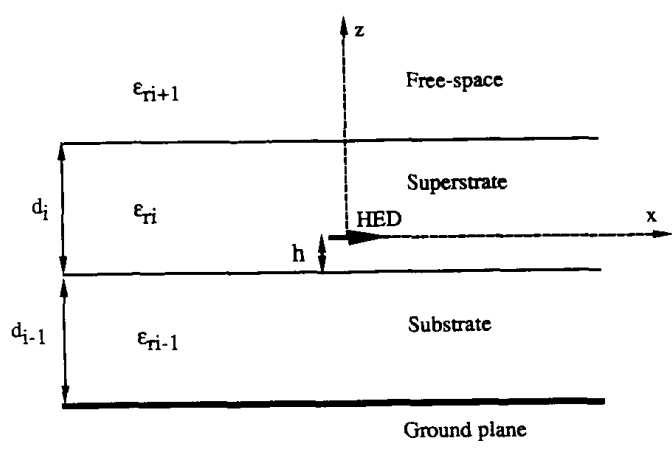

Fig. 1. Substrate-superstrate geometry.

However, recently a novel approach to circumventing this problem has been developed [4],[5], one that employs closedform expressions for the spatial domain Green's functions corresponding to the vector and scalar potentials associated with a horizontal electric dipole (HED) located over a thick substrate. This technique has been further extended [6],[7] to microstrip geometries with both a substrate and a superstrate, whose thicknesses can be arbitrary. The closed-form Green's functions have been used in a method of moments formulation to analyze planar microstrip structures and substantial savings in computation time has been realized [8]. In this paper, we briefly discuss the derivation of these closed-form Green's functions and then employ them to address the problem of spurious radiation calculation from microstrip interconnects, modeled herein as single or double microstrip lines terminated by complex loads. Though the numerical results presented in this paper pertain to microstrip etches in printed circuit boards with substrates only, the method of analysis applies equally well to the case where a superstrate is also present, as for instance in Fig. 1.

\section{Derivation of Closed-Form GreEN'S FUNCTIONS FOR A GENERAL MICROSTRIP GEOMETRY}

One of the most commonly used numerical techniques for solving electromagnetic problems is the MoM, which is based upon the transformation of an operator equation into a matrix equation. Although the MoM is preferred over differential equation methods for the microstrip circuit and radiation problems, because it is relatively efficient in terms of computation time, it is still quite time-consuming due to the slow convergence of the integrals involved. In view of this, it would be instructive to demonstrate the difficulties that one might face in the application of the conventional moment method approach to microstrip geometries before presenting 
the derivation of the closed-form Green's functions for the vector and scalar potentials. It is well known that the MoM can be applied either in the spatial domain [9] or in the spectral domain [10], although the latter is more suitable for microstrip geometries. Both approaches will be examined here from the point of view of computational efficiency.

\section{A. Conventional Method of Moments (MoM)}

Consider a general microstrip structure at $z=0$ plane and assume that the substrate layer extends to infinity in the transverse directions ( $x y$-plane). Let the thickness and the permittivity of the substrate be denoted by $d_{i-1}$ and $\varepsilon_{r i-1}$, respectively.

The tangential electric fields on the plane of the patch $(z=0)$ can be written in terms of the Green's functions for the vector and scalar potentials, $G_{s s(s=x, y)}^{A}$ and $G_{q}$, respectively, as

$$
\begin{aligned}
& E_{x}=-j \omega G_{x x}^{A} * J_{x}+\frac{1}{j \omega} \frac{\partial}{\partial x}\left[G_{q} * \nabla \cdot \boldsymbol{J}\right] \\
& E_{y}=-j \omega G_{y y}^{A} * J_{y}+\frac{1}{j \omega} \frac{\partial}{\partial y}\left[G_{q} * \nabla \cdot \boldsymbol{J}\right]
\end{aligned}
$$

where $*$ denotes spatial convolution; and $J$ is the surface current density on the conducting etches.

Using the basis functions $J_{x n}$ and $J_{y n}$, and the testing functions $T_{x m}$ and $T_{y m}$, and following the MoM procedure one obtains the following equations for the unknown coefficients $A_{n}$ and $B_{n}$ of the expansion functions $J_{x n}$ and $J_{y n}$,:

$$
\begin{aligned}
\sum_{n} A_{n} & \left\{\left\langle T_{x m}, G_{x x}^{A} * J_{x n}\right\rangle\right. \\
+ & \left.\frac{1}{\omega^{2}}\left\langle T_{x m}, \frac{\partial}{\partial x}\left[G_{q} * \frac{\partial}{\partial x} J_{x n}\right]\right\rangle\right\} \\
& +\sum_{n} B_{n}\left\{\frac{1}{\omega^{2}}\left\langle T_{x m}, \frac{\partial}{\partial x}\left[G_{q} * \frac{\partial}{\partial y} J_{y n}\right]\right\rangle\right\}=0 \\
\sum_{n} B_{n}\left\{\left\langle T_{y m}, G_{y y}^{A} * J_{y n}\right\rangle\right. & \left.+\frac{1}{\omega^{2}}\left\langle T_{y m}, \frac{\partial}{\partial y}\left[G_{q} * \frac{\partial}{\partial y} J_{y n}\right]\right\rangle\right\} \\
+ & \sum_{n} A_{n}\left\{\frac{1}{\omega^{2}}\left\langle T_{y m}, \frac{\partial}{\partial y}\left[G_{q} * \frac{\partial}{\partial x} J_{x n}\right]\right\rangle\right\}=0 .
\end{aligned}
$$

The first inner product term in (3) is written below as an example

$$
\begin{aligned}
\left\langle T_{x m}, G_{x x}^{A} * J_{x n}\right\rangle= & \int_{D(T)} \int d x d y T_{x m}(x, y) \\
& \cdot \int_{D(B)} \int d x^{\prime} d y^{\prime} \\
& \cdot G_{x x}^{A}\left(x-x^{\prime}, y-y^{\prime}\right) J_{x n}\left(x^{\prime}, y^{\prime}\right)
\end{aligned}
$$

where $D(T)$ and $D(B)$ represent the domain of the testing and expansion functions, respectively, and

$$
G_{x x}^{A}(\rho)=\frac{1}{4 \pi} \int_{-\infty}^{+\infty} d k_{\rho} k_{\rho} H_{o}^{(2)}\left(k_{\rho} \rho\right) \tilde{G}_{x x}^{A}\left(k_{\rho}\right) .
$$

The evaluation of the inner products in (6) is very timeconsuming because of the oscillatory nature and the slow convergence behavior of the integrands of the Green's functions.

An alternative approach to solving the current distribution problem using MoM is to work in the spectral domain instead, where the necessary Green's functions can be expressed in closed forms. Nevertheless, the integrals for the matrix elements now span over infinite ranges and can be highly oscillatory in nature [11]. As a consequence, these integrals are still very expensive to evaluate and this prompts us to look for ways by which this process could either be accelerated or circumvented.

\section{B. Closed-Form Green's Functions for the Vector and ScalarPotentials}

One remedy for the aforementioned convergence problem is to express the spatial domain Green's functions in closed forms so that the inner products become two-dimensional integrals over a finite range, and the time-consuming part of the moment method in the spatial domain, which is the evaluation of the Green's functions, is completely avoided. The Sommerfeld integrals for the Green's functions corresponding to the vector and scalar potentials are written as

$$
G^{A, q}=\frac{1}{4 \pi} \int_{\mathrm{SIP}} d k_{\rho} k_{\rho} H_{o}^{(2)}\left(k_{\rho} \rho\right) \tilde{G}^{A, q}\left(k_{\rho}\right)
$$

where

$\tilde{G}^{A}$ the Green's function of the vector potential in the spectral domain,

$\tilde{G}^{q}$ the Green's function of the scalar potential in the spectral domain,

$H_{o}^{(2)}$ the Hankel function of the second kind,

SIP the Sommerfeld integration path (see [11]).

The procedure for deriving closed-form expressions for the Green's functions entails the following steps:

1) Obtain the Green's functions for the vector and scalar potentials in the spectral domain.

2) Find the quasi-static images (real images) and their contributions by using the Sommerfeld identity.

3) Find the surface-wave poles and calculate their contributions analytically.

4) Approximate the remaining integrand, which is now a smooth and relatively rapidly decaying function of the integration variable $k_{\rho}$ in (7), in terms of complex exponentials, say by using the least-square Prony or the pencil-of-function method.

The derivation of the closed-form Green's functions for the vector and scalar potentials for a horizontal electric dipole located at the interface between the substrate and superstrate, as shown in Fig. 1, has been presented in some detail in [6] 
and [7], and will be omitted here. We merely give below the closed-form expressions for the pertinent Green's functions

$$
\begin{aligned}
& G_{A}^{x x}=\frac{\mu}{4 \pi} \frac{e^{-j k_{i} r_{d}}}{r_{d}}-\frac{\mu}{4 \pi} \frac{e^{-j k_{i} r_{i 1}}}{r_{i 1}}+\frac{\mu}{4 \pi}(-j 2 \pi) \sum_{i=1}^{N_{\mathrm{TE}}} k_{\rho p(i)} \\
& H_{o}^{(2)}\left(k_{\rho p(i)} \rho\right) \operatorname{Res} 1^{(i)}+\frac{\mu}{4 \pi} \sum_{i=1}^{N_{1}} b_{1 i} \frac{e^{-j k_{i} r_{1 i}}}{r_{1 i}} \\
& G_{q}=\frac{1}{4 \pi \varepsilon_{i}} \frac{e^{-j k_{i} r_{d}}}{r_{d}} \\
& +\frac{K_{i, i+1}}{4 \pi \varepsilon_{i}} \frac{e^{-j k_{i} r_{i 2}}}{r_{i 2}}-\frac{K_{i, i+1} K_{i-1, i}}{4 \pi \varepsilon_{i}} \frac{e^{-j k_{i} r_{i 3}}}{r_{i 3}} \\
& -\frac{K_{i-1, i}}{4 \pi \varepsilon_{i}} \frac{e^{-j k_{i} r_{i 4}}}{r_{i 4}}-\frac{\left(1-K_{i-1, i}^{2}\right)}{4 \pi \varepsilon_{i}} \frac{e^{-j k_{i} r_{i 5}}}{r_{i 5}} \\
& -\frac{K_{i, i+1} K_{i-1, i}}{4 \pi \varepsilon_{i}} \frac{e^{-j k_{i} r_{i 6}}}{r_{i 6}} \\
& -\frac{j}{2 \varepsilon_{i}} \sum_{i=1}^{N_{\mathrm{TE}}+N_{\mathrm{TM}}} k_{\rho p(i)} H_{o}^{(2)}\left(k_{\rho p(i)} \rho\right) \operatorname{Res} 2^{(i)} \\
& +\frac{1}{4 \pi \varepsilon_{i}} \sum_{i=1}^{N_{2}} b_{2 i} \frac{e^{-j k_{i} \boldsymbol{r}_{2 i}}}{r_{2 i}}
\end{aligned}
$$

where $K_{i, i+1}=\left(\varepsilon_{r i}-\varepsilon_{r i+1}\right) /\left(\varepsilon_{r i}+\varepsilon_{r i+1}\right), K_{i-1, i}=\left(\varepsilon_{r i-1}-\right.$ $\left.\varepsilon_{r i}\right) /\left(\varepsilon_{r i-1}+\varepsilon_{r i}\right) ; N_{\mathrm{TE}}$ and $N_{\mathrm{TM}}$ are the numbers of TE and TM surface-wave poles $k_{\rho p(i)} ; N_{1}$ and $N_{2}$ are the numbers of complex images for the vector and scalar potentials, respectively; $\left(b_{1 i}, r_{1 i}\right)$ and $\left(b_{2 i}, r_{2 i}\right)$ are the coefficient and complex distance pairs of the complex images for the vector and scalar potentials, respectively; and

$$
\begin{aligned}
r_{d} & =\sqrt{\rho^{2}+z^{2}} ; \quad r_{i 1}=\sqrt{\rho^{2}+\left(z+2 h+2 d_{i-1}\right)^{2}} \\
r_{i 2} & =\sqrt{\rho^{2}+\left(2 d_{i}-2 h-z\right)^{2}} ; \quad r_{i 3}=\sqrt{\rho^{2}+\left(2 d_{i}+z\right)^{2}} \\
r_{i 4} & =\sqrt{\rho^{2}+(z+2 h)^{2}} ; \\
r_{i 5} & =\sqrt{\rho^{2}+\left(2 d_{i-1}+z+2 h\right)^{2}} \\
r_{i 6} & =\sqrt{\rho^{2}+\left(2 d_{i}-z\right)^{2}}
\end{aligned}
$$

For further details and explanations of notation, the reader is referred to [6] and [7] cited above.

We consider an example of a microstrip geometry for which the relative dielectric constant and the substrate thickness are 4.0 and $0.0203 \mathrm{~cm}$ ( $=8 \mathrm{mils}$ ), respectively. The frequency of operation is chosen to be $1 \mathrm{GHz}$. For these parameters, the substrate thickness is 0.00135 in terms of the wavelength in the medium and there exists only one TM surface-wave pole located at $k_{\rho}=0.20944$. In this example, we have used two and four complex images for the closed-form representations of the Green's functions for the vector and scalar potentials, respectively, i.e., have chosen $N_{1}=2$ and $N_{2}=4$ in (8) and (9). As can be seen from Figs. 2 and 3, the closed-form Green's functions and the numerically integrated Sommerfeld integrals are found to be in very good agreement up to a moderate distance between the observation and source points, beyond which the closed-form approximation begins to deviate

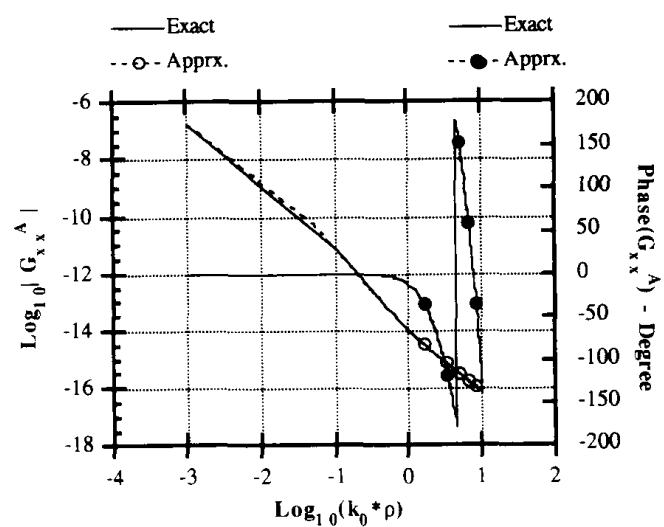

Fig. 2. The Green's function for the vector potential; magnitude and phase. $\hat{c}_{r i-1}=4.0 . \varepsilon_{r i}=1.0, d_{i-1}=0.02032 \mathrm{~cm}$, freq $=1.0 \mathrm{GHz}$.

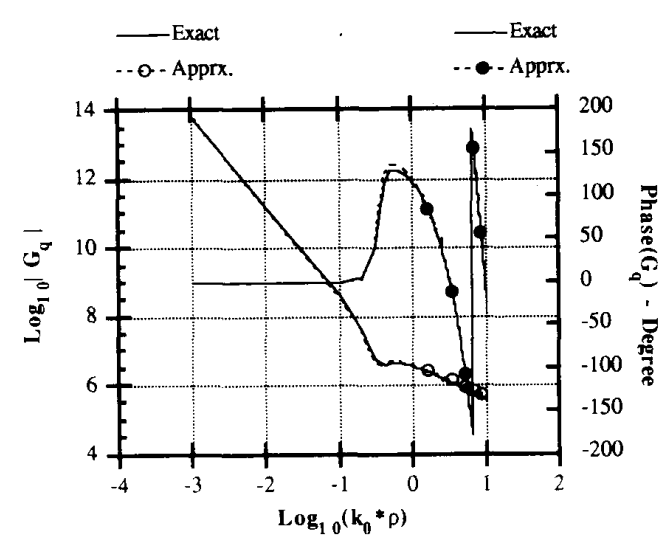

Fig. 3. The Green's function for the scalar potential; magnitude and phase $\varepsilon_{r i-1}=4.0 \varepsilon_{r i}=1.0 . d_{i-1}=0.02032 \mathrm{~cm}$, frequency $=1.0 \mathrm{GHz}$.

somewhat from the exact Green's functions. The Sommerfeld integrals themselves were evaluated using accurate quadrature schemes after the surface wave poles were extracted and their contributions evaluated analytically.

It is possible to employ far-field approximations [12] to derive a more accurate representation of the Green's functions for large $\rho$. However, this is often unnecessary because the size of the conductors on a substrate is usually on the order of a wavelength or less, and the closed-form representations of the Green's functions are usually adequate.

It should be noted that the procedure given above is quite general and is applicable to a wide range of frequencies and material parameters. And yet, its use results in savings of computation time in the calculation of the Green's functions as well as in the application of the MoM to microstrip problems.

Before closing this section, we would like to mention that small argument approximations of the Sommerfeld integrals have been introduced recently by Mosig and Gardiol [13]. However, it is believed that the closed-form Green's functions presented here have a wider range of applicability, needed in the present analysis, than could be realized by using the Mosig and Gardiol expressions. 


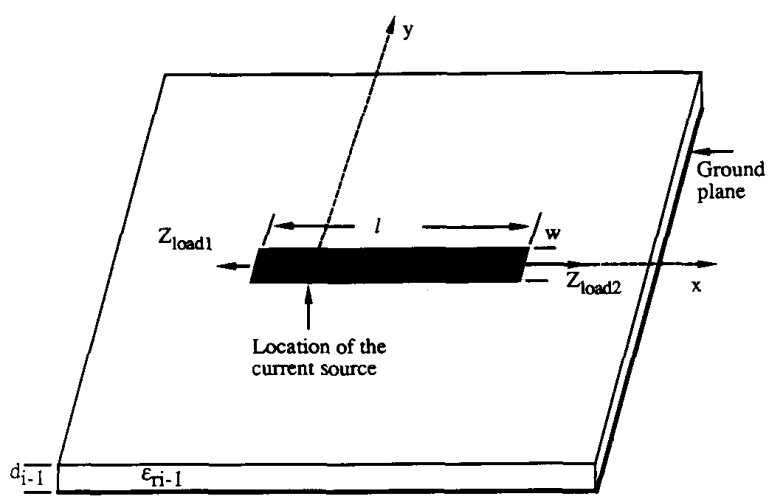

Fig. 4. Geometry of a microstrip line terminated by complex loads

\section{Microstrip Line Terminated By COMPLEX LoADS}

In this section, we investigate the problem of spurious radiation from a microstrip etch by considering the geometry of a microstrip line on a substrate as shown in Fig. 4. Since our ultimate goal is to access the level of spurious radiation from an integrated circuit, we start with the calculation of the radiated power from an interconnect. The interconnect is modeled here as a microstrip line that is fed by a current source and terminated by complex loads at both ends. The location of the current source is arbitrary as are the complex load impedances terminating the line. Our objective is to compute the current distribution on the line and the level of spurious radiation as a function of the length of the line and the load impedances.

\section{A. Current Distribution on a Microstrip Line Terminated by Complex Loads}

Fig. 4 shows the geometry of a microstrip line fed by an arbitrarily located current source and terminated by complex impedances at both ends. The substrate is assumed to be infinitely wide in the $x$ - and $y$-directions, to have a thickness $d_{i-1}$, and a relative permittivity $\varepsilon_{r i-1}$.

The electric field along the line ( $x$-direction) are given in (1) in terms of the current densities $J_{x}$ and $J_{y}$. For this problem, the $y$-component of the current density is assumed to be negligible because the width of the microstrip line $w$ is much smaller than the wavelength in the dielectric medium. Therefore, only the $x$-directed current density is represented in terms of the expansion functions, expressed as

$$
J_{x}(x, y)=\sum_{n} I_{n} J_{x n}(x, y)+J_{s}(x, y)
$$

where $I_{n}$ is the unknown coefficient of the expansion function, and $J_{s}$ is the expansion function for the current source. By substituting (10) into (1) and testing the resulting equations using the expansion functions $J_{x m}$ (Galerkin's method of moments), the following algebraic equation is obtained for the coefficients $I_{n}$ for each $m$ :

$$
\sum_{n} I_{n}\left[\left\langle J_{x m}, G_{x x}^{A} * J_{x n}\right\rangle+\frac{1}{\omega^{2}}\left\langle J_{x m}, \frac{\partial}{\partial x}\left(G_{q} * \frac{\partial}{\partial x} J_{x n}\right)\right\rangle\right]
$$

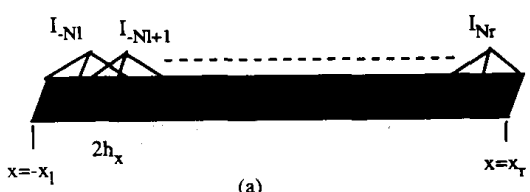

(a)
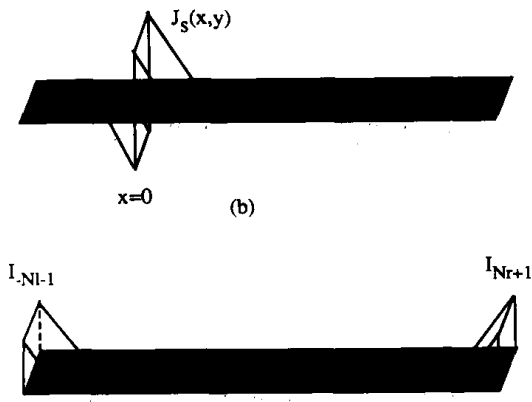

(c)

Fig. 5. Expansion functions representing the current density (a) on the line, (b) at the source, and (c) at the load terminals.

$$
=-\left\langle J_{x m}, G_{x x}^{A} * J_{s}\right\rangle-\frac{1}{\omega^{2}}\left\langle J_{x m}, \frac{\partial}{\partial x}\left(G_{q} * \frac{\partial}{\partial x} J_{s}\right)\right\rangle .
$$

If the number of equations, i.e., $m$, is less than the number of unknowns $n$, additional equations must be provided in order to realize a square matrix and to obtain a unique solution for the coefficients $I_{n}$.

The expansion functions, apart from those that represent the source and load currents, are chosen to be rooftops, which are triangular functions in the longitudinal direction, uniform in the transverse direction, and are defined mathematically as

$$
J_{x m}(x, y)= \begin{cases}\frac{1}{w}\left[1-\frac{\left|x-m h_{x}\right|}{h_{x}}\right], & |y| \leq 2 / w \\
& \begin{array}{l}
(m-1) h_{x} \leq x \leq \\
(m+1) h_{x}
\end{array} \\
0, & \text { elsewhere }\end{cases}
$$

where $h_{x}$ is the support of the expansion functions (see Fig. 5).

The source and load contributions to the current density on the microstrip line are taken into account by employing suitable expansion functions for them and relating them to the other equations. The expansion functions for the current distribution associated with the source and loads are given by

$$
J_{s}(x, y)=\left\{\begin{array}{ll}
\frac{-1}{w}\left(1+\frac{x}{h_{x}}\right), & -h_{x} \leq x \leq 0,|y| \leq \frac{w}{2} \\
\frac{1}{w}\left(1-\frac{x}{h_{x}}\right), & 0 \leq x \leq h_{x},|y| \leq \frac{w}{2} \\
0, & \text { elsewhere }
\end{array}\right\}
$$




$$
J_{\text {Load }}(x, y)=\left\{\begin{array}{lc}
-\frac{1}{w} \frac{x+N_{1} h_{x}}{h_{x}}, & -\left(N_{1}+1\right) h_{x} \leq x \\
\frac{1}{w} \frac{x-N_{r} h_{x}}{h_{x}}, & N_{r} h_{x} \leq x \\
& \leq\left(N_{r}+1\right) h_{x},|y| \leq \frac{w}{2} \\
& |y| \leq \frac{w}{2} \\
0, & \text { elsewhere }
\end{array}\right.
$$

and plotted in Fig. 5(b) and 5(c), respectively. The source basis functions model the excitation of the microstrip line by a coaxial cable with its center conductor attached to the etch whose width is much smaller than the wavelength. These expansion functions have been chosen to be compatible with the rooftops (12), which have already been employed to represent the current density on the microstrip line. However, these functions are piecewise continuous while the rooftops are piecewise differentiable functions. Consequently, the integrals corresponding to the expansion functions of the source and loads in (11) would be divergent unless some physical requirements are imposed upon them to render them convergent [14]. The problem of piecewise continuous functions as expansion functions for the current density is the discontinuity that gives rise to infinite, nonphysical charge density, i.e., a singularity in its derivative that needs to be inserted in (11). Once this singularity is removed, a process that can be justified on physical principles of conservation of charge at the source and load terminals, the integrals involving these expansion functions become convergent.

Upon choosing the expansion functions given above, and deleting the nonphysical singularities caused by the divergence of the current, it becomes possible to justify the step of integration by parts and to transfer the derivatives in front of the convolution integrals in (11) over to the functions $J_{x m}$. Equation (11) can then be rewritten as

$$
\begin{aligned}
& \sum_{n=-N_{1}-1}^{N_{r}+1} I_{n}\left[\left\langle J_{x m}, G_{x x}^{A} * J_{x n}\right\rangle\right. \\
& \left.-\frac{1}{\omega^{2}}\left\langle\frac{\partial}{\partial x} J_{x m},\left(G_{q} * \frac{\partial}{\partial x} J_{x n}\right)\right\rangle\right] \\
& =-\left\langle J_{x m}, G_{x x}^{A} * J_{s}\right\rangle+\frac{1}{\omega^{2}}\left\langle\frac{\partial}{\partial x} J_{x m},\left(G_{q} * \frac{\partial}{\partial x} J_{s}\right)\right\rangle \\
& m=-N_{1}, \cdots, N_{r}
\end{aligned}
$$

where the number of equations $\left(N_{1}+N_{r}+3\right)$ exceeds the number of unknowns $\left(N_{1}+N_{r}+1\right)$ by two. However, as detailed in the next section, two additional equations can be obtained by enforcing the necessary boundary conditions at the load terminals.

Each inner product term in (14) is a four-dimensional integral provided that the closed-form Green's functions are used. Since the numerical integration of a four-dimensional integral is quite expensive, even though the integration is over a finite domain, the convolution between the Green's function and the expansion functions is transferred over to the two expansion functions involved in each term. If the expansion functions have been chosen such that their convolution can be performed analytically, which is the case for the choice of the expansion functions given in (12), (13a), and (13b), the inner products in (14) reduce to double integrals over finite domains.

\section{B. Additional Equations for the Load Expansion Functions}

In order to relate the coefficients of the load expansion functions to the rest of the expansion functions, we need to invoke two additional boundary conditions at the load terminals. Since the product of the load impedance and the line current is the voltage difference between the line and the ground, the voltages at the load terminals can be expressed in terms of the load impedances, the coefficients of the load expansion functions, and the coefficients of the other expansion functions defined on the microstrip line. Next, a simple ${ }^{1}$ and computationally efficient approach, based on transmission line analysis, can be used to relate the load impedances to the surface current density on the line. In transmission line analysis, it is well known that the total voltage $V(x)$ and the total current $I(x)$ on the line are related by the following first-order differential equations:

$$
\frac{d I(x)}{d x}=-Y V(x) ; \quad \frac{d V(x)}{d x}=-Z I(x)
$$

where $Y=j \beta / Z_{0}$ and $Z=j \beta Z_{0}$ are the shunt admittance and series impedance per unit length of the line, respectively. The characteristic impedance $Z_{0}$ and propagation constant $\beta$ of the line are calculated by using empirical formulas based on a quasi-static analysis [15]. If the derivatives in (15) are approximated by finite differencing and the resulting equations are related to each other for the load terminals at $x=x_{1}$ and $x=x_{r}$, the following equations are obtained [see Fig. 5(a) and $5(\mathrm{c})]$ :

$$
\begin{aligned}
& \left(1+j \beta h_{x} \frac{Z_{L 1}}{Z_{0}}-\frac{\beta^{2} h_{x}^{2}}{2}\right) I_{-N_{1}-1}-I_{-N_{1}}=0 \\
& -I_{N_{r}}+\left(1+j \beta h_{x} \frac{Z_{L r}}{Z_{0}}-\frac{\beta^{2} h_{x}^{2}}{2}\right) I_{N_{r}+1}=0
\end{aligned}
$$

where $V\left(-x_{1}\right) / I\left(-x_{1}\right)=-Z_{L 1}$ and $V\left(x_{r}\right) / I\left(x_{r}\right)=Z_{L r}$ are employed. Note that (16) and (17) are dependent upon the finite-difference approximation. By supplementing (14) with these two terminal conditions and solving for $I_{n}$ using matrix methods, the current distribution on the microstrip line terminated by the complex load impedances $Z_{L 1}$ and $Z_{L r}$ is obtained. The results thus obtained are presented in the next section and are compared with those derived by using a simpler transmission line approach.

\section{Spurious Radiation}

After solving for the current distribution on the microstrip line, whether by using the TL approach or the MoM, both the near- and far-field components can be calculated by using appropriate Green's functions. The spurious radiated power can then be obtained by integrating the Poynting vector over

\footnotetext{
'The question of using a more rigorous approach has been discussed in [8]
} 
a closed surface, which could be chosen as a rectangular box enclosing the microstrip line.

In this paper, the spurious radiated power is defined as the total power crossing a plane parallel to the plane of the substrate. This power is expressed as

$P=\frac{1}{2} \operatorname{Re} \int_{S} \int d s \cdot \boldsymbol{E} \times \boldsymbol{H}^{*} \cong \frac{1}{2} \operatorname{Re} \int_{S} \int d x d y\left(E_{x} H_{y}^{*}-E_{y} H_{x}^{*}\right)$

Since the calculation of the field components requires a convolution integral for each expansion function that is used to represent the current density on the microstrip line, implementing (18) in the spatial domain becomes computationally expensive. Therefore, the field components in (18) are transformed into the spectral domain, and the total power is expressed in the spectral domain as

$$
\begin{aligned}
& P=\frac{1}{2} \operatorname{Re}\{ \frac{1}{4 \pi^{2}} \int_{-\infty}^{\infty} d k_{x} d k_{y} \\
&\left.\cdot\left[\left(\tilde{G}_{x x}^{E} \tilde{J}_{x}\right)\left(\tilde{G}_{y x}^{H} \tilde{J}_{x}\right)^{*}-\left(\tilde{G}_{y x}^{E} \tilde{J}_{x}\right)\left(\tilde{G}_{x x}^{H} \tilde{J}_{x}\right)^{*}\right]\right\}
\end{aligned}
$$

where the Green's functions for the electric and magnetic fields are obtained by using the immittance approach [16].

\section{Results and Discussions}

In this section, we present a few representative numerical results for the current distribution and spurious radiated power from microstrip etches. The following parameters have been chosen for the examples given below: the dielectric constant of the medium $\varepsilon_{r i-1}=4.0$; the ratio of the width of the microstrip line $w$ to the thickness $d_{i-1}$ of the substrate $=4.0$; the thickness of the substrate $d_{i-1}=8.0 \mathrm{mils}(0.0203 \mathrm{~cm})$; and the frequency of operation $=1.0 \mathrm{GHz}$. The source is located approximately $1.0 \mathrm{~cm}$ from the left edge of the microstrip line and has an amplitude of 2 Amps.

The current distribution on a line can often be predicted intuitively for standard terminations e.g., a match, open circuit, or short circuit. This prompts us to use these cases as examples of our calculations. Excellent agreement is observed between the current distributions calculated by the TL approach (see [7] for details) and the MoM for a microstrip line terminated at both ends by matched loads, open or short circuits, excepting in the vicinity of the resonance for the last two cases [7], [8]. This behavior is attributable to the difference in the resonant lengths of the line predicted by the MoM and the $\mathrm{TL}$ approaches. As an example of a complex termination, we have chosen a resistance of $20 \mathrm{~K}$ in parallel with a 8 $\mathrm{pF}$ capacitance, which represents the typical input impedance of a TTL gate. The current distributions for this termination have been calculated by using both the TL and the MoM approaches, and are exhibited in Fig. 6. It is observed that the current calculated by the TL approach is slightly different from that of the MoM, because, as mentioned above, the length of the line is close to the resonant length for this load termination.

A study of the current distributions for different lengths of the line leads us to conclude that in general, the TL approach predicts the current distribution reasonably well, provided that

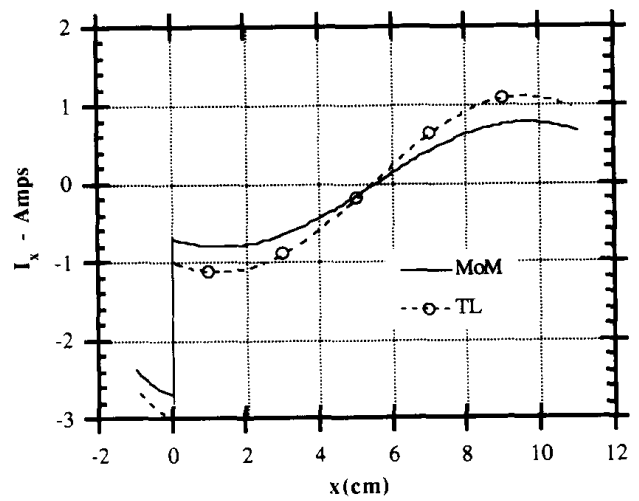

Fig. 6. Magnitude of current distribution obtained by using MoM and TL approaches for a complex load termination $Z_{L}=(20 \mathrm{~K} / 8 \mathrm{pF})$. Discontinuity at $x=0$ is due to the attachment of the current source.

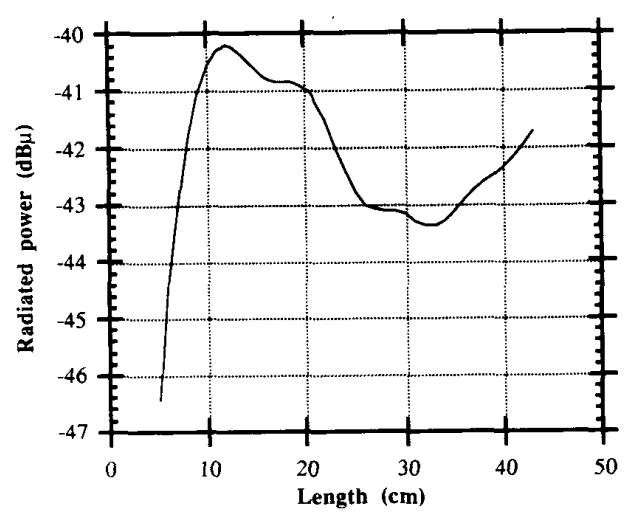

Fig. 7. Radiated power as a function of the length of the line for matched termination at both ends.

the frequency of operation is not too close to the resonant frequency of the resonator represented by the truncated line.

The spurious power, as defined by (19), is calculated for: 1) a microstrip line terminated by matched loads; 2 ) open circuits at both terminals; and 3) a matched load on the left and a complex load of $8 \mathrm{pF} / 20 \mathrm{~K} \Omega$ on the right terminal. For the power calculations, the source current amplitude is assumed to be $1 \mathrm{~mA}$. The results for the radiated power are shown in Figs. 7-9, respectively. As seen from Fig. 7, the radiation from a microstrip line terminated by a pair of matched loads is very small in comparison to those for other loads. The highest radiation occurs for the open-circuited transmission lines of resonant length, for which the radiated power reaches the level of $1.0 \mu \mathrm{W}$ for a $1 \mathrm{~mA}$ source current (see Fig. 8). The radiated power has a sharp peak around the resonance length of the line, and it becomes essentially negligible for off-resonance lengths. Fig. 9 shows that for the combination of matched and complex load terminations, the total radiated power is slightly larger than that of matched load termination case (Fig. 7).

The spurious radiated powers for open and short circuit terminations have been measured for test boards and are shown in Fig. 10(a) and (b). For measurement purposes it was more 


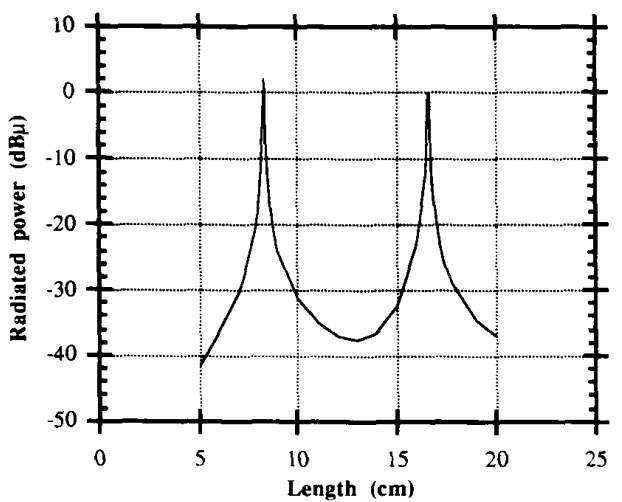

Fig. 8. Radiated power as a function of the length of the line for open-circuit termination at both ends.

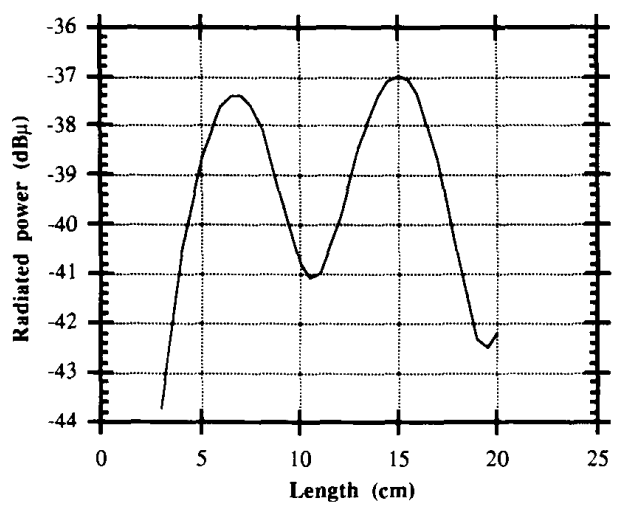

Fig. 9. Radiated power as a function of the length of the line for a matched load and a complex load $Z_{L}=(20 \mathrm{~K} / 8 \mathrm{pF})$ terminations.

convenient to investigate the variation of the radiated power as a function of frequency and to choose the dimension of the etch as follows: length $=7.6 \mathrm{~cm}$; width $=0.22 \mathrm{~cm}$; substrate $\varepsilon_{r}=2.62$; thickness of substrate $=0.0794 \mathrm{~cm}$. Also, the measured data was taken only in the principal plane and normalized at one frequency before comparing them with the calculated data that assumed an input current of $1 \mathrm{~mA}$. We note that the frequency variations of the radiated power agree quite well with the calculated results, especially for the open-circuit case, which is easier to realize in practice.

\section{ASYMMETRIC PARALLEL MICROSTRIP LINES TERMINATED BY ARBITRARY COMPLEX LOADS}

Coupled planar transmission lines with equal line lengths have been extensively investigated in the past two decades by using quasi-static approaches [17], and full-wave analyses [18], [19]. The quasi-static approximations avoid extensive numerical calculations required in the conventional full-wave analysis, but often do not provide sufficiently accurate results for radiation problems.

Parallel microstrip etches can be used either as coupled lines or as interconnects for digital circuits. As interconnects, they

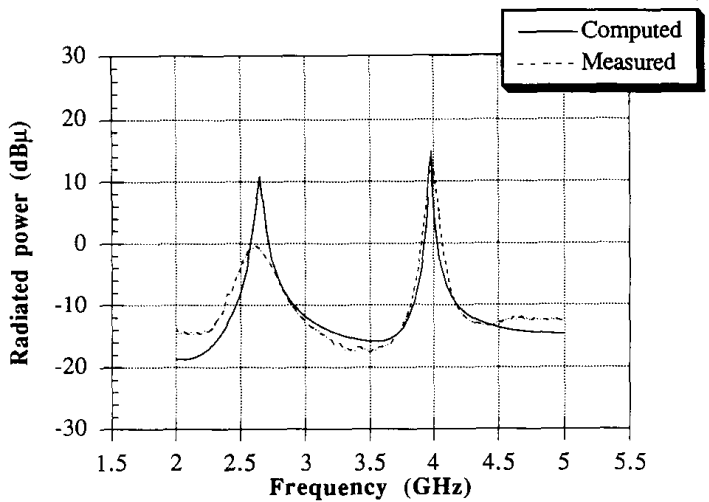

(a)

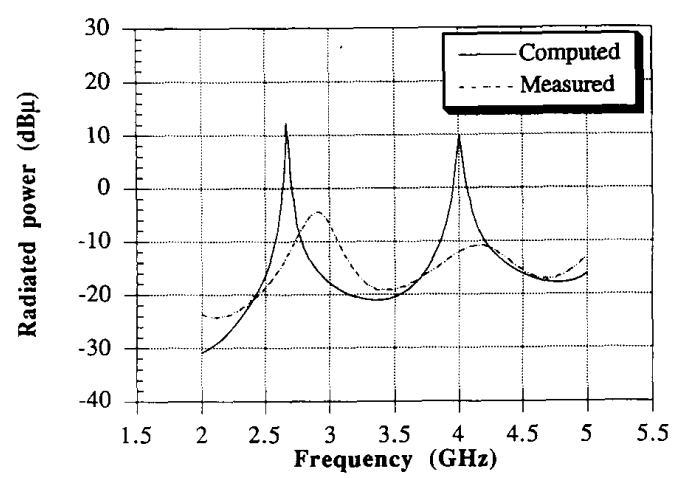

(b)

Fig. 10. Comparison of numerical and experimental (normalized) results for spurious radiated power as a function of frequency. Line length $=7.6 \mathrm{~cm}$; width $=0.22 \mathrm{~cm} ; \varepsilon_{r}=2.62$; substrate thickness $=0.079 \mathrm{~cm}$. (a) open circuit at both ends; (b) short circuit at both ends.

may, in general, have different lengths and be terminated by different complex loads. Therefore, in this work, we investigate the problem of determining the current distribution and radiation leakage from parallel microstrip edges, which consist of two parallel microstrip lines with arbitrary lengths and terminations. Since the lengths of the lines can be different, their relative positions can be adjusted arbitrarily as long as they remain parallel to each other.

\section{A. Formulation of the Problem}

The geometry of an asymmetric parallel microstrip lines is shown in Fig. 11, where line -1 is fed by an arbitrarily located current source through the substrate. The lengths and widths of the lines are denoted by $l_{1}, l_{2}$ and $w_{1}, w_{2}$, respectively; the center-to-center spacing and the gap between the two lines are defined as the parameters $\Delta y$ and $s$, respectively; $\Delta x$ defines the amount of the shift in the longitudinal direction. The substrate thickness is $d_{i-1}$, and the relative permittivity is $\varepsilon_{r i-1}$.

As in the case of a single line described in Section III-A, the $y$-component of the current density is assumed to be negligible while the $x$-component of the current density is expanded in 


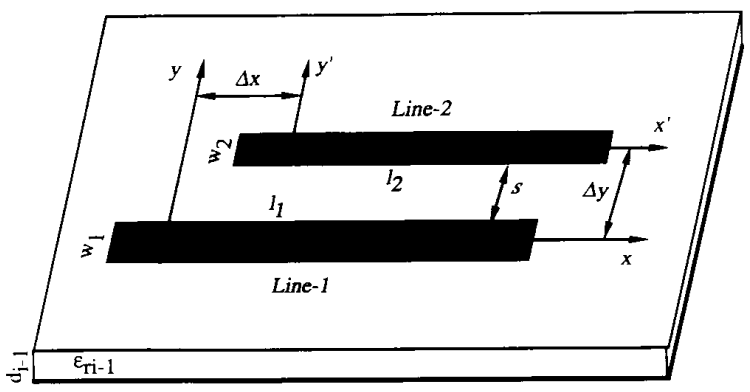

Fig. 11. Geometry of asymmetric parallel lines.

terms of the expansion functions as

$J_{x}(x, y)=\sum_{n} I_{1 n} J_{1 x n}(x, y)+J_{s}(x, y)+\sum_{n} I_{2 n} J_{2 x n}(x, y)$

where $I_{1 n}$ and $I_{2 n}$ are the unknown coefficients of the expansion functions used on the active and passive lines, respectively; and $J_{i x n}$ and $J_{s}$ are the expansion functions for the current density on the line and at the source terminal, respectively, whose mathematical representations have been given in (12) and (13). By following the same procedure as described in Sections II-A and III-A, simultaneous equations for the unknown coefficients of the expansion functions can be obtained. However, for the two-line case, the number of resulting equations is less than the number of unknowns by four (in contrast to two for the single-line case). As before, we impose the boundary conditions at the load terminals to obtain the four requisite equations involving the complex load terminations. The applications of the load boundary conditions are discussed in detail in the next section.

\section{B. Imposing the Boundary Conditions at the Load Terminals}

We now discuss an extension of the procedure given in Section III-B that leads to the four supplementary equations needed to obtain a unique solution for the current distributions on the coupled-line system. In general, the geometry of the two etches under consideration is asymmetric. Thus, each of the four terminals of the parallel line system may be regarded as belonging to a single- or coupled-line system depending upon the relative length of the passive line and the longitudinal shift $\Delta x$. For instance, if $-x_{l 1}<-x_{l 2}+\Delta x$ and $x_{r 1}<x_{r 2}+\Delta x$, then the terminals -1 and -4 are treated as though they are terminals of single lines, to be handled separately, while the terminals -2 and -3 are associated with the coupled line system.

The differential equations for a coupled-line system are given by

$$
\begin{aligned}
& -\frac{d V_{1}(x)}{d x}=j \omega L_{11} I_{1}(x)+j \omega L_{12} I_{2}(x) \\
& -\frac{d V_{2}(x)}{d x}=j \omega L_{21} I_{1}(x)+j \omega L_{22} I_{2}(x) \\
& -\frac{d I_{1}(x)}{d x}=j \omega C_{11} V_{1}(x)+j \omega C_{12} V_{2}(x) \\
& -\frac{d I_{2}(x)}{d x}=j \omega C_{21} V_{1}(x)+j \omega C_{22} V_{2}(x)
\end{aligned}
$$

where the $L_{i j}$ 's and $C_{i j}$ 's are the Maxwellian inductances and capacitances per unit length of the coupled line, which can be calculated by using a quasi-static approach [20].

By introducing the finite-difference approximation in (21), we obtain the following desired supplementary terminal conditions:

$$
\begin{gathered}
I_{1(-N 11-1)}\left[1+j \beta_{1} h_{x} \frac{Z_{L 11}}{Z_{01}}-\frac{\beta_{1}^{2} h_{x}^{2}}{2}\right]-I_{1(-N 11)}=0 \\
\frac{C_{22}}{j \omega h_{x} \Delta_{c}} I_{1(N r 1)}-\left[\frac{C_{22}}{j \omega h_{x} \Delta_{c}}+Z L r 1+j \omega h_{x} L_{11}\right] \\
\cdot I_{1(N r 1+1)}-\frac{C_{12}}{j \omega h_{x} \Delta_{c}} I_{2(m-1)} \\
+\left[\frac{C_{12}}{j \omega h_{x} \Delta_{c}}-j \omega h_{x} L_{11}\right] I_{2 m}=0
\end{gathered}
$$

$$
\begin{gathered}
{\left[\frac{C_{21}}{j \omega h_{x} \Delta_{c}}-j \omega h_{x} L_{21}\right] I_{1 m}-\frac{C_{21}}{j \omega h_{x} \Delta_{c}} I_{1(m+1)}} \\
-\left[\frac{C_{11}}{j \omega h_{x} \Delta_{c}}+Z_{L 12}+j \omega h_{x} L_{22}\right] I_{2(-N 12-1)} \\
+\frac{C_{11}}{j \omega h_{x} \Delta_{c}} I_{2(-N 12)}=0 \\
-I_{2(N r 2)}+I_{2(N r 2+1)}\left[1+j \beta_{2} h_{x} \frac{Z_{L 12}}{Z_{02}}-\frac{\beta_{2}^{2} h_{x}^{2}}{2}\right]=0
\end{gathered}
$$

where $\beta_{1}, Z_{01}$ and $\beta_{2}, Z_{02}$ are the propagation constants and the characteristic impedances of the isolated active and passive lines, respectively; and $\Delta_{c}=C_{11} C_{22}-C_{12} C_{21}$.

The current distributions and the spurious radiated power for coupled lines are presented in the next section.

\section{Results and Discussions}

The following parameters have been chosen for the examples given below: the dielectric constant of the medium $\varepsilon_{r i-1}=4.0$; the thickness of the substrate $d_{i-1}=8.0 \mathrm{mils}$ $(0.0203 \mathrm{~cm})$; the widths of the lines $w=w_{1}=w_{2}=4 d_{i-1}$; and the frequency of operation $f=1.0 \mathrm{GHz}$. The location of the source is chosen to be $0.4 \mathrm{~cm}$ away from the left edge of line -1 , i.e., $x_{11}=0.4 \mathrm{~cm}$, unless stated otherwise.

The current distribution on a coupled line system, for which both the active and passive lines have equal lengths $(\Delta x=0)$, and all terminated by an open circuit at all the load terminals, is obtained for some typical spacings $s(=\Delta y-w)$ between the lines. The current distributions on the active and passive lines are given in Fig. 12 for two different spacings between the lines (the source current is still 2 Amps as before). Although the typical spacing between the lines is equal to the width of the lines, we have chosen the spacing $s$ equal to half and twice the width of the lines, in order to illustrate the effect of the spacing on the coupling. As expected, when the spacing between the lines is large compared to the thickness of the substrate, the coupling to the passive line is very small, and 


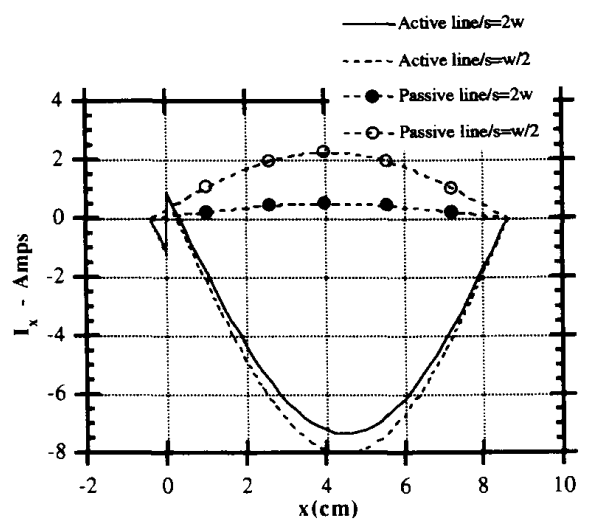

Fig. 12. Current distribution on a coupled line terminated by open circuits. length $=9.0 \mathrm{~cm}, f=1.0 \mathrm{GHz}, \varepsilon_{r i-1}=4.0, d_{i-1}=8.0 \mathrm{mils}$ $u_{1}=u_{2}=32$ mils.

there is little effect on the current distribution on the active line. However, it is observed that the coupling to the passive line and the change in the current distribution on the active line are more pronounced when the lengths of the lines are close to the resonant length

To complete the study of the coupled-line interconnect system, we investigate complex impedances that represent typical terminations for interconnects used in digital circuits. In this example, the active and passive lines, which are separated by the distance $w / 2$, are terminated by matched loads on the left terminals and complex impedances of 20$\mathrm{k} \Omega$ resistance paralleled with $8-\mathrm{pF}$ capacitance on the right terminals. The magnitude and phase of the current distribution on the coupled line with the specified terminations is given in Fig. 13, which shows that the active line seems to have a resonance behavior even though one of the terminations is matched to the characteristic impedance of the single line

The spurious radiated power has been calculated, for an input current of $1 \mathrm{~mA}$, for the coupled-line system terminated by open circuits, matched loads, and complex impedances. These are presented in Figs. 14-16, respectively. For the open-circuit terminations, the level of the spurious radiation from a single microstrip line that has the same dimensions as the coupled lines is also given in Fig. 14 for the sake of comparison. The spurious radiation is lower than $-30 \mathrm{~dB} \mu$ for most of the lengths of the lines when terminated by open circuits; however it goes up significantly, to $2-4 \mathrm{~dB} \mu$, at around the resonant lengths. The radiated power increases with the length of the lines and saturates at the level of $-40 \mathrm{~dB} \mu$ for the matched load terminations, which is less than the amount of radiation at the off-resonance lengths from the coupled line with open-circuit terminations.

In general, the microstrip lines that are used as interconnects may have different lengths and their starting and end terminals may be shifted with respect to each other, such lines are designated here as asymmetric parallel lines. Fig. 17 gives the current distribution on asymmetric parallel microstrip lines terminated by open circuits at all four terminals. Each line is $8.0 \mathrm{~cm}$ long, shifted by $4.0 \mathrm{~cm}$, and the spacing $s$ is equal

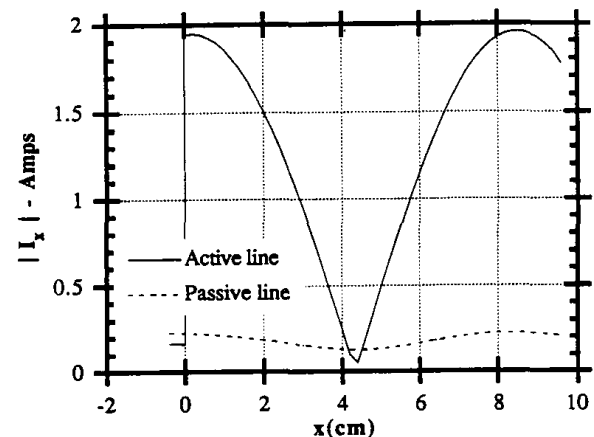

(a)

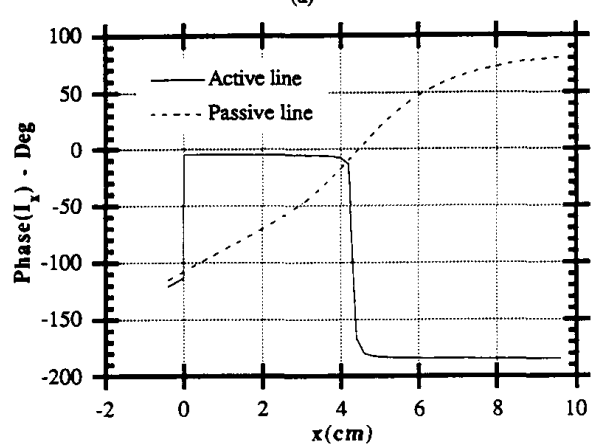

(b)

Fig. 13. Current distribution on a coupled line; (a) Magnitude, (b) Phase $Z_{L 11}=Z_{L 12}=Z_{0} \cdot Z_{L r 1}=Z_{L r^{2}}=(20 \mathrm{~K} \Omega / 8 \mathrm{pF}) \cdot f=1 \mathrm{GHz}$ $\hat{\varepsilon}_{r_{i-1}}=4.0 . d_{i-1}=8 \mathrm{mils}, w_{1}=w_{2}=32 \mathrm{mils}, s=16 \mathrm{mils}$

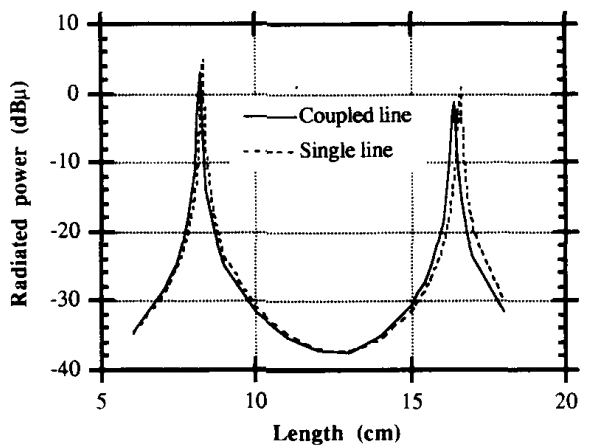

Fig. 14. Radiated power as a function of the length of the single and coupled lines for open circuit termination at all terminals. $f=1.0 \mathrm{GHz}$. $\bar{\varepsilon}_{r i-1}=4.0, d_{i-1}=8.0 \mathrm{mils}, w_{1}=w_{2}=32 \mathrm{mils}, s=32 \mathrm{mils}$.

to the line width $w$. Note that the current distributions on the active and passive lines are in phase, and this causes an increase in the level of spurious radiation compared to that from a single line with the same dimensions as the active line. To assess the effect of the passive line on the level of radiation, the power of spurious radiation is calculated as a function of the length of the passive line while the length of the active line is kept constant at $8.0 \mathrm{~cm}$, as shown in Fig. 18. For comparison purposes, we note, by referring to Fig. 8 , that a single microstrip line of $8.0 \mathrm{~cm}$ in length and terminated by open circuits at both ends radiates around $-15.3 \mathrm{~dB} \mu$. In 


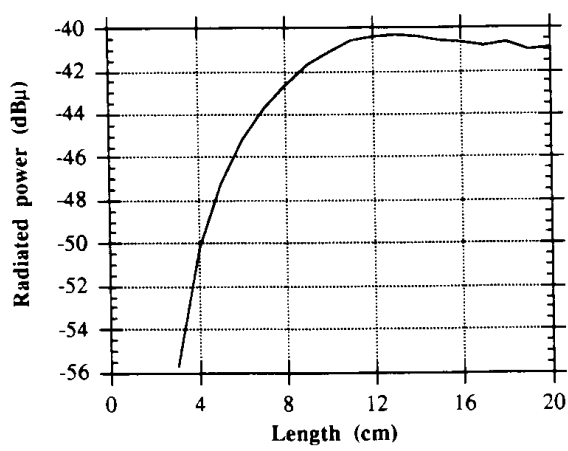

Fig. 15. Radiated power as a function of the length of the coupled line for matched load termination at all terminals. $f=1.0 \mathrm{GHz}$, $\varepsilon_{r i-1}=4.0 . d_{i-1}=8.0$ mils, $w_{1}=w_{2}=32$ mils, $s=32$ mils.

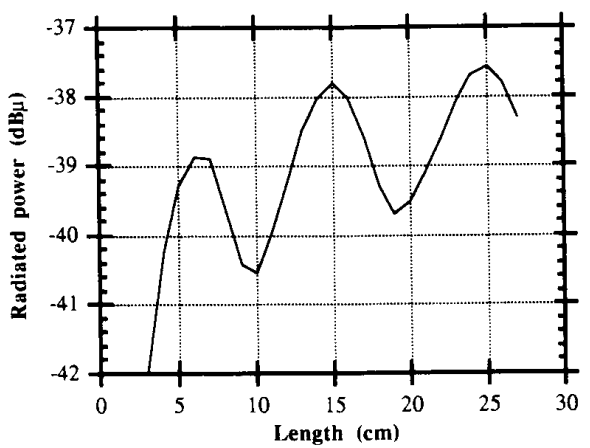

Fig. 16. Radiated power as a function of the length of the coupled line. $Z_{L 11}=Z_{L 12}=Z_{0}, Z_{L r 1}=Z_{L r 2}=(20 \mathrm{~K} \Omega / 8 \mathrm{pF}) . f=1.0 \mathrm{GHz}$ $\varepsilon_{r i-1}=4.0 . d_{i-1}=8.0$ mils, $w_{1}=w_{2}=32 \mathrm{mils}, s=16$ mils

the vicinity of the resonance, the level of radiation increases up to $-9.5 \mathrm{~dB} \mu$ as the length of the passive line approaches the resonant length due to the in-phase coupling, and goes down to $-20 \mathrm{~dB} \mu$ as soon as the coupling is out of phase. However, for off-resonance lengths, the level of radiation is almost equal to that from a single microstrip line. Another example is given for an asymmetric line system terminated by a matched load on the left and the complex load $(20 \mathrm{k} \Omega / 8 \mathrm{pF})$ on the right terminals of the active line, while the terminals on the passive line are left open-circuited. The spurious radiation from this geometry is presented in Fig. 19, where the length of the passive line is used as an independent variable.

\section{CONCLUSIONS}

In this work, spatial domain Green's functions for a general class of microstrip geometries have been presented in closed forms and have been used to analyze single and coupled microstrip lines terminated by complex loads.

The use of the closed-form spatial domain Green's functions in the method of moments formulation reduces the computation time significantly as compared to the conventional formulation carried out in the spectral domain. For instance, in a numerical experiment with 40 rooftop expansion functions, the computation time for the current distribution is on the order

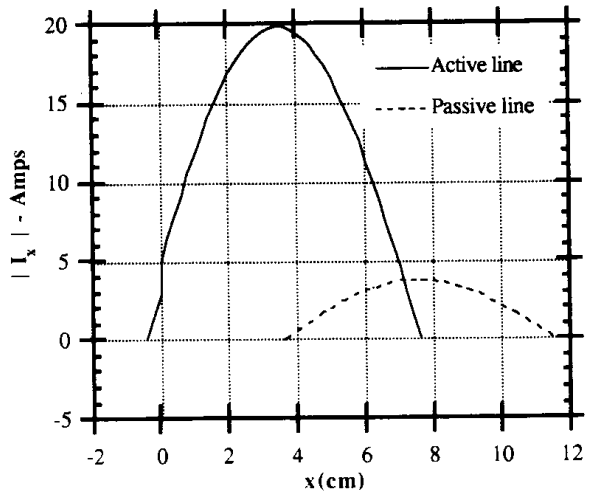

Fig. 17. Current distribution on asymmetric parallel lines for open circuit terminations at all terminals. $f=1.0 \mathrm{GHz}, \Sigma_{r i-1}=4.0, d_{i-1}=8.0 \mathrm{mils}$, $u_{1}=w_{2}=32$ mils, $s=32$ mils, $l_{1}=8.0 \mathrm{~cm}, l_{2}=8.0 \mathrm{~cm}, \Delta x=4.0 \mathrm{~cm}$.

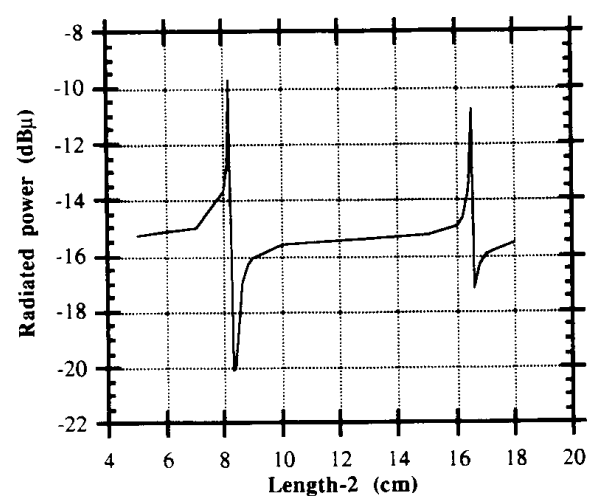

Fig. 18. Radiated power from asymmetric parallel lines for open circuit terminations at all terminals. $f=1.0 \mathrm{GHz}, \varepsilon_{r i-1}=4.0 . d_{i-1}=8.0 \mathrm{mils}$, $w_{1}=u_{2}=32$ mils, $s=32$ mils, $l_{1}=8.0 \mathrm{~cm}, \Delta x=4.0 \mathrm{~cm}$.

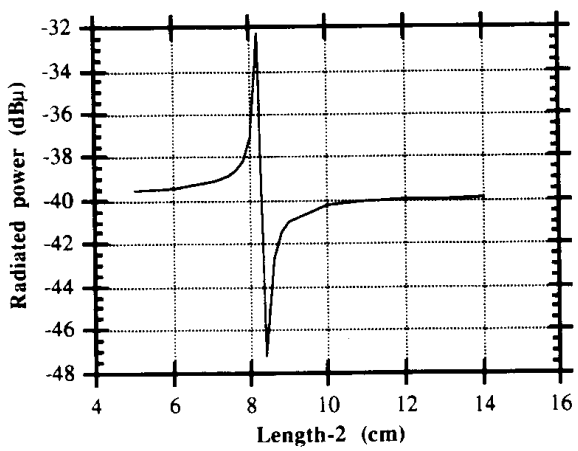

Fig. 19. Radiated power from asymmetric parallel lines $Z_{L 11}=Z_{0}, Z_{L r 1}$ $=(20 \mathrm{k} \Omega / 8 \mathrm{pF}) \cdot Z_{L \prime 2}=Z_{L \cdot 2}=$ open-circuited, $f=1.0 \mathrm{GHz}$ $\varepsilon_{r-1}=4.0 . d_{i-1}=8.0$ mils, $w_{1}=w_{2}=32$ mils, $s=32$ mils, $l_{1}=8.0$ $\mathrm{cm}, \Delta x=4.0 \mathrm{~cm}$

of 1-min CPU time on DEC station 5100 when the closed-form Green's functions are used, whereas it takes on the order of 10-20 min on the Cray/YMP for the same calculation using the spectral domain moment method in conjunction with an acceleration technique. 
The investigation of the radiation leakage from a microstrip line terminated by complex loads has shown that the highest radiation occurs when the length of the line is near resonance, and the terminations are open circuits. It was also found that by using a passive line parallel to the active one can reduce the radiation significantly, provided that the coupling is out of phase. This information could be potentially useful to a designer engaged in the planning of the layout of a lowradiation module for a digital circuit.

The frequency variation of the spurious radiated power was validated by experimental measurements.

\section{ACKNOWLEDGMENT}

The authors wish to thank Dr. J. E. Schutt-Aine for providing the capacitance and inductance matrices of the coupled lines that were used in the computations reported in this paper. Support from the National Center for Supercomputing Applications at the University of Illinois for computer time on the Cray/YMP supercomputer is also acknowledged.

\section{REFERENCES}

[1] R. K. Hoffmann, Handbook of Microwave Integrated Circuits. Dedham, MA: Artech House, 1987.

[2] Fred E. Gardiol, "Radiation from microstrip circuits: An introduction," Int. J. Microw. Millim.-Wave Comput.-Aided Eng., vol. 1, no. 2, pp. 225-235, 1991 .

[3] A Sommerfeld, Partial Differential Equations in Physics. New York Academic, 1949

[4] Y. L. Chow, J. J. Yang, D. H. Fang, and G. E. Howard, "Closed-form spatial Green"s function for the thick substrate," IEEE Trans. Microwave Theory Tech., vol. 39, pp. 588-592, Mar. 1991.

[5] D. G. Fang, J. J. Yang, and G. Y. Delisle, "Discrete image theory for horizontal electric dipoles in a multilayered medium," IEE Proc., vol. 135, part H, Oct. 1988.

[6] M. I. Aksun and R. Mittra, "Derivation of closed-form Green's functions for a general microstrip geometry," IEEE Trans. Microwave Theory Tech., Nov. 1992, scheduled for publication.

[7] M. I. Aksun and R. Mittra, "Investigation of radiation characteristics of microstrip etches," University of Illinois at Urbana-Champaign, Interim Tech. Rep. UILU-ENG-92-2210, Mar. 1992.

[8] M. I. Aksun and R. Mittra, "Estimation of spurious radiation from microstrip etches using closed-form Green"s functions," IEEE Trans. Microwave Theors Tech., Nov. 1992, scheduled for publication.

[9] R. F. Harrington, Field Computation by Moment Methods. New York: MacMillan, 1983.

[10] T. Itoh and R. Mittra, "Spectral domain approach for calculating the dispersion characteristics of microstrip line," IEEE Trans. Microwave Theor. Tech.. vol. MTT-21, pp. 496-499, 1973.

111] A. Kouki. "Application of the spectral domain technique to discontinuity and spurious radiation problems in microwave circuits," $\mathrm{Ph} . \mathrm{D}$. dissertation, University of Illinois at Urbana-Champaign, 1991.
[12] S. Barkeshli, P. H. Pathak, and M. Marin, "An asymptotic closedform microstrip surface Green's function for the efficient moment method analysis of mutual coupling in microstrip antennas," IEEE Trans. Antennas Propagat., vol. 38, pp. 1374-1383, Sept. 1990.

[13] Mosig and Gardiol, "A dynamic radiation model for microstrip structure," Advances in Electronics and Electron Physics, vol. 59, pp. $139-237,1982$.

[14] M. I. Aksun and R. Mittra, "Choices of basis and testing functions for the method of moments in electromagnetic problems," Submitted for publication.

[15] H. Sobol, "Application of integrated circuit technology to microwave frequencies," Proc. IEEE, vol. 59, pp. 1200-1211, Aug. 1971.

[16] T. Itoh, "Spectral domain immittance approach for dispersion characteristics of generalized printed transmission lines," IEEE Trans. Microwave Theory Tech., vol. MTT-28, pp. 733-736, July 1980.

[17] T. G. Bryant and J. A. Weiss, "Parameters of microstrip transmission lines and of coupled pairs of microstrip lines," IEEE Trans. Microwave Theory Tech., vol. MTT-16, pp. 1021-1027, Dec. 1968.

[18] C. H. Chan and R. Mittra, "Analysis of MMIC structures using an efficient iterative approach," IEEE Trans. Microwave Theory Tech., vol. MTT-36, pp. 96-105, Jan. 1984.

[19] C. Wei, R. F. Harrington, J. R. Mautz and T. Sarkar, "Multiconductor transmission lines in multilayered dielectric media," IEEE Trans. Microwave Theory Tech, vol. MTT-32, pp. 439-450, April 1984.

[20] J. E. Schutt-Aine, "Modeling and simulation of high-speed digital circuit interconnects," Ph.D. dissertation, University of Illinois at UrbanaChampaign, 1988.

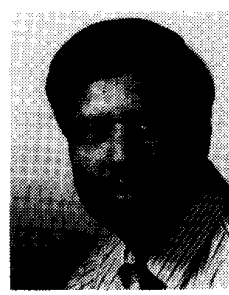

Raj Mittra (S'54-M'57-SM'69-F'71) is the Director of the Electromagnetic Communication L.aboratory of the Electrical and Computer Engineering Department and Research Professor of the Coordinated Science Laboratory at the Liniversity of Illinois. Ho is a Past-President of $A P$-S and has served as the coditor of the II:III: IRANSACTIONS ON ANTRNAS ANI PROPACiNTION. Ile won the Guggenheim lellowship Award in 196.5 and the IEFEE Centennial Medal in 1984. He has been a Visiting Professor at Oxford Lniversity, Oxford, England, and at the Technical Lniversity of Denmark, L.ynghy, Denmark. Currently, he serves as the North American editor of the Journal AEU. He is President of RM Associales, which is a consulting organization providing services to several industrial and govemmental organizations.

IIis professional interests include the areas of computational electromagnetics, electromagnetic modeling of electronic packaging, radar scattering, satellite antennas, microwave and millimeter wave integrated circuits, frequency selective surfaces, EMP and E.MC analysis, and remote sensing. He has published approximately 350 journal papers and 22 books or book chapters on various lupics related to electromagnetics 